\title{
Virus-induced gene silencing and virus- induced flowering in strawberry (Fragaria $\times$ ananassa) using apple latent spherical virus vectors
}

\author{
Chunjiang Li ${ }^{1}$, Noriko Yamagishi ${ }^{2}$, Ichiro Kasajima ${ }^{2}$ and Nobuyuki Yoshikawa (i] ${ }^{1,2}$
}

\begin{abstract}
Apple latent spherical virus (ALSV) vector is a convenient alternative to genetic transformation in horticultural plants, especially in species recalcitrant to genetic transformation. ALSV, an RNA virus, can infect a wide variety of plant species including major horticultural plants without inducing symptoms. Here, methodologies were developed for infection of ALSV vectors to strawberry seedlings and plantlets cultured in vitro. A seed-propagated $F_{1}$ hybrid strawberry cultivar 'Yotsuboshi' was aseptically grown on half-strength Murashige-Skoog medium for 1 month and true leaves were inoculated with an ALSV RNA preparation by particle bombardment. ALSV vector infection rates varied from 58 to 100\% according to the insertion sequences, in 'Yotsuboshi' seedlings. Plantlets ('Dover') propagated in vitro could also be infected with ALSV vector at a similar infection rate. For virus-induced gene silencing (VIGS), we prepared an ALSV vector carrying a 201 nucleotide segment of the strawberry phytoene desaturase gene. 'Yotsuboshi' and 'Dover' plants infected by this vector generated completely white leaves at fifth or sixth true leaves and above. For virus-induced flowering (VIF), we used an ALSV vector expressing the Arabidopsis thaliana flowering locus T gene. Strawberry seedlings infected by this vector started to flower from about 2 months post inoculation and bore fruits with viable seeds. The ALSV vector was no longer detected in any of the seedlings from early-flowered strawberries. Thus, the ALSV vector may be beneficial for examination of gene functions by VIGS in strawberry, and VIF using ALSV vector constitutes an effective new plant breeding technique for the promotion of cross-breeding in strawberry.
\end{abstract}

\section{Introduction}

Strawberry (Fragaria $\times$ ananassa) is a popular crop worldwide with production exceeding four million tons per year ${ }^{1}$. Despite the long cultivation history of strawberries from the fiteenth century, strawberry breeding remains a major concern of farmers and researchers with regard to a variety of traits such as fruit quality (taste), fruit colour, fruit firmness, fruit storability, ascorbic acid

Correspondence: Nobuyuki Yoshikawa (yoshikawa@iwate-u.ac.jp)

${ }^{1}$ Faculty of Agriculture, Iwate University, Morioka 3-18-8, Iwate 020-8550, Japan ${ }^{2}$ Agri-Innovation Research Center, Iwate University, Morioka 3-18-8, Iwate 0208550, Japan

These authors contributed equally: Chunjiang Li, Noriko Yamagishi, Ichiro Kasajima. content, everblooming habit, disease resistance and heat/ cold resistance ${ }^{2}$. Changes in these agronomically important traits are expected to be caused by mutations in genomic DNA. For example, the everblooming (continuous flowering) trait of woodland strawberry (Fragaria vesca) is caused by a 2 bp deletion in the coding region of the KSN gene ${ }^{3}$, a strawberry homologue of the Terminal Flower 1 (TFL1) gene. The genetic mapping approach has also identified the $\mathrm{FaOMT}$ gene mutation as the regulator of variation in the release of mesifurane, one of the volatiles of strawberry fruit, based on complete cosegregation of the identified 30-bp mutation in the FaOMT promoter ${ }^{4}$.

\section{(c) The Author(s) 2019}

(c) (i) Open Access This article is licensed under a Creative Commons Attribution 4.0 International License, which permits use, sharing, adaptation, distribution and reproduction cc. in any medium or format, as long as you give appropriate credit to the original author(s) and the source, provide a link to the Creative Commons license, and indicate if changes were made. The images or other third party material in this article are included in the article's Creative Commons license, unless indicated otherwise in a credit line to the material. If material is not included in the article's Creative Commons license and your intended use is not permitted by statutory regulation or exceeds the permitted use, you will need to obtain permission directly from the copyright holder. To view a copy of this license, visit http://creativecommons.org/licenses/by/4.0/. 
Specific genetic mutations causing changes in other agronomically important traits in strawberry remain mostly unknown, although candidate genes are being suggested through experimental efforts such as gene expression analyses ${ }^{5-8}$. The release of genomic sequences and reports of thousands of DNA markers to detect polymorphisms on the chromosomes are also expected to greatly accelerate both forward and reverse genetic studies of strawberry ${ }^{9}$. Such knowledge with regard to agronomically important genes and their mutations both informs basic plant biology and accelerates strawberry breeding based on DNA information ${ }^{10}$.

Following identification of agronomically important or candidate genes, their characterization by genetic overexpression or suppression represents a standard strategy for confirmation of their functions. The protocol for genetic transformation of strawberry by Agrobacterium tumefaciens was first established in $1990^{11,12}$. Whereas the transformation efficiency is generally around 5\%, the efficiency may be increased up to $100 \%^{13-16}$, although transformation rate appears to depend on the strawberry cultivar. Specifically, 100\% transformation efficiency was achieved in an everbearing (day-neutral) cultivar 'Calypso', using the super-virulent Agrobacterium strain AGLO ${ }^{14}$.

Transient expression/suppression by infiltration of Agrobacterium into strawberry fruits has also been utilized for rapid analysis of gene functions. In this case, Agrobacterium causes overexpression or RNA interference of the target gene ${ }^{17,18}$, depending on the nucleotide sequence introduced into the transfer DNA region of the binary plasmid. The target strawberry gene may also be suppressed via virus-induced gene silencing (VIGS) using tobacco rattle virus (TRV) vectors ${ }^{19,20}$. Considering that genetic transformation typically requires 15 months from the start of the experiments to the production of strawberry fruits ${ }^{18}$, these transient systems enable much faster estimation of gene function than stable transformation, although the genes are expressed/suppressed only in sections within strawberry fruits (more strictly, receptacles). Alternatively, genes can be expressed/suppressed in whole strawberry fruits when Agrobacterium is repeatedly injected into fruits at least three times ${ }^{18}$. Such transient systems are currently utilized for the analysis of gene functions with regard to strawberry fruit phenotypes, such as pigmentation, aroma generation, ripening and disease resistance ${ }^{21}$. Genes can also be transiently expressed in strawberry leaves by infiltration of Agrobacterium for the analysis of their functions ${ }^{22}$.

Once agronomically important mutations in the genome are identified, they can be combined by crossbreeding and DNA marker selection. However, the long generation time of crops often constitutes a major problem for efficient cross-breeding. Although the genomes of vegetatively propagated cultivars/crops such as apple and pear remain genetically heterozygous, short generation time is also important when the crop genome is homogenized for establishment of seed-propagated genetically homozygous cultivars/crops and $F_{1}$ hybrid cultivars/crops, such as rice and maize. Thus, in addition to controlling growth conditions such as cultivation in greenhouse or incubator, high $\mathrm{CO}_{2}$ levels, tiller removal, paclobutrazol treatment, grafting on rootstock, and embryo rescue $^{23-26}$, transgenic expression of the leafy or flowering locus $T$ (FT) gene or suppression of the TFL1 gene also comprise important techniques for inducing early flowering and reducing generation time ${ }^{25,27,28}$.

Virus-induced flowering (VIF) can also be effective for reducing the generation time of crops. In VIF, crops are infected with RNA virus vectors expressing an $F T$ gene to induce early flowering ${ }^{29}$. An advantage of VIF is that the genomic DNA of crops is not transformed, and the infected transgenic virus is rarely carried to the progeny (next-generation) plants. In addition, virus infection does not depend on the specific crop cultivar in many cases. Rather, infectivity of virus vectors depends on the host range of viruses; thus, zucchini yellow mosaic virus has been used for the expression of Arabidopsis thaliana FT $(A t F T)$ in squash/pumpkin (Cucurbita moschata) ${ }^{30}$, cotton leaf crumple virus was used for AtFT expression in $\operatorname{cotton}^{31}$ and citrus leaf blotch virus was used for AtFT or citrus $F T$ expression in citrus plants ${ }^{32}$ for the successful induction of early flowering. As apple latent spherical virus (ALSV) exhibits a relatively wide host range, in previous studies we used an ALSV vector for the expression of $A t F T$ or other $F T$ genes such as a gentian $F T$ to induce early flowering in $A$. thaliana, tobacco (Nicotiana) plants, soybean, apple, petunia, lisianthus (eustoma) and gentian ${ }^{33-37}$. The progeny plants of crossbred gentian generated by using ALSV-based VIF were confirmed for elimination of the virus, cleared governmental review and started to be grown in an open field in Hachimantai City of Japan for selection of a new cultivar. This appears to be the first example of the application of any New Plant Breeding Technique in Japan.

Again, ALSV enjoys advantages as a virus vector: ALSV has a wide host range, latently infects most plant species (without viral symptoms) and evenly infects the upper leaves. ALSV may provide novel high-throughput methods for estimation of the functions of strawberry genes, and promotion of cross-breeding by shortening the generation time of strawberry. To examine the utility of this technique, in the present study we inoculated ALSV vectors to a commercially important cultivar of strawberry. As a result, the abilities of the ALSV vector to successfully infect strawberry seedlings and to spread to every organ of the shoot, as well as to induce VIGS and VIF in strawberry plants, were ascertained. 


\section{Results}

\section{Conditions of ALSV inoculation}

As ALSV vectors have not yet been tested in strawberry, we first aimed to identify a successful condition of ALSV inoculation to strawberry. ALSV consists of RNA1 and RNA2 genomes, with each genome encoding a single polyprotein. For comparison of inoculation conditions, ALSV vector without any insertion of external sequence (wild-type; wtALSV) was used (Fig. 1a). Because ALSV does not necessarily infect horticultural plants easily, ALSV is usually inoculated as viral RNA instead of via rub inoculation or agroinfiltration. For example, gold particles coated with viral RNA are bombarded by gene guns to cotyledons of germinating seeds in the case of apple ${ }^{33,36}$, or to true leaves of juvenile seedlings in the case of gentian $^{35,38}$.

In the present study, we first attempted bombardment of RNA-coated gold particles to true leaves of strawberry seedlings (a seed-propagated cultivar 'Yotsuboshi') grown on soil in a plastic plug tray (Fig. 1b). As strawberries had developed three or four true leaves at this stage (1 month after sowing), gold particles were shot at two expanded true leaves. Although the amount of RNA per shot $(7 \mu \mathrm{g})$ in this trial was greater than the standard amount $(5 \mu \mathrm{g})$ for apple and gentian, and two shots were taken per each leaf, reverse transcription-polymerase chain reaction (RTPCR) analysis performed on upper leaves at 1 month post inoculation revealed that none of the 16 inoculated strawberry plants was infected by wtALSV (infection rate of $0 \%$; Table 1). Using wtALSV, we also inoculated strawberry seedlings grown aseptically on half-strength Murashige-Skoog (MS) medium with RNA-coated gold particles, under the same conditions as the plants grown in the plug tray. RT-PCR analysis was performed at 1 month post inoculation, and eight out of nine inoculated plants were infected by wtALSV (infection rate of $89 \%$; Table 1). Fig. 1d shows the DNA bands obtained by RT-PCR analysis of strawberry seedlings grown aseptically on half-strength MS medium. The wtALSV was detected both in inoculated leaves and upper leaves of strawberry plants, except for the \#3 plant in which ALSV was not detected in the upper leaf. Inoculation of wtALSV to in vitro culture of 'Dover' resulted in successful infection at an infection rate of $63 \%$. Thus, seedlings and in vitro cultures grown aseptically were inoculated in the following experiments.

\section{VIGS in strawberry leaf}

Next, we tested whether ALSV could be used for VIGS in strawberry leaves. In a previous report, fragmental phytoene desaturase (PDS) genes of tobacco (tPDS), watermelon (cuPDS) and soybean (soyPDS) were successfully used for VIGS by using ALSV vectors, and whitening of leaves (photo-bleaching) was observed in tobacco (Nicotiana tabacum), Nicotiana benthamiana, Nicotiana glutinosa, Nicotiana occidentalis, cucumber (Cucumis sativus), pumpkin (Cucurbita pepo), watermelon (Citrullus lanatus), luffa (Luffa cylindrica), bottle gourd (Lagenaria siceraria), soybean (Glycine max), pea (Pisum sativum), adzuki bean (Vigna angularis) and cowpea (Vigna unguiculata) ${ }^{39}$. The strawberry PDS gene (accession no. FJ795342) (designated FaPDS) encodes a protein of 568 amino acid residues with the protein sequence being highly homologous to tPDS, cuPDS and soyPDS, suggesting that FaPDS functions as phytoene desaturase in strawberry.

Clear photo-bleaching by VIGS of PDS genes by ALSV vectors is almost always observed when the fragment sequences of the PDS genes are 200 nucleotides (nt) or longer $^{39}$. In the present study, a $201 \mathrm{nt}$ fragment of FaPDS was synthesized artificially and then introduced into the XhoI-SmaI-BamHI (XSB) cloning site of the RNA2 vector of ALSV (Fig. 1a). Viral RNA prepared from this clone possesses an additional 201nt insertion between MP and Vp25 of the RNA2 genome (Fig. 2a). This virus was designated ALSV-FaPDS. An RNA preparation from leaves infected with ALSV-FaPDS was bombarded on true leaves of strawberry seedlings ('Yotsuboshi') aseptically grown on half-strength MS medium. The result indicated that all 12 strawberry plants inoculated with this virus were infected (infection rate of $100 \%$; Table 1). Photobleaching was also observed in the majority of each upper leaf and leaf petiole of the plants infected by ALSV-FaPDS (Fig. 2b). Here, partial photo-bleaching was observed in 'close' upper leaves, which expanded immediately after inoculation, and overall photo-bleaching was observed in 'remote' upper leaves, which expanded later than 'close' upper leaves. Overall photo-bleaching of leaves was observed at least 1 month after inoculation. Conversely, photo-bleaching was never observed in the plants infected by wtALSV (Fig. 2b). A cultivar 'Dover' propagated from in vitro culture also showed a clear photo-bleaching phenotype of newly developed leaves after inoculation of ALSV-FaPDS (Fig. 2c). These results suggest that ALSV vector can be effectively used for the analysis of gene function of strawberry.

\section{VIF of strawberry}

An ALSV vector carrying full-length AtFT inserted at the XSB site was also used in the present study. This vector, ALSV-AtFT, expresses AtFT as a part of the polyprotein encoded by the RNA2 genome of ALSV (Fig. 3a). After translation of the polyprotein, AtFT is excised from the polyprotein through digestion with protease. RNA of ALSV-AtFT was bombarded onto the true leaves of 1-month-old, aseptically grown seedlings in vitro. A total of 12 plants were inoculated in this analysis, of which seven were systematically infected by 


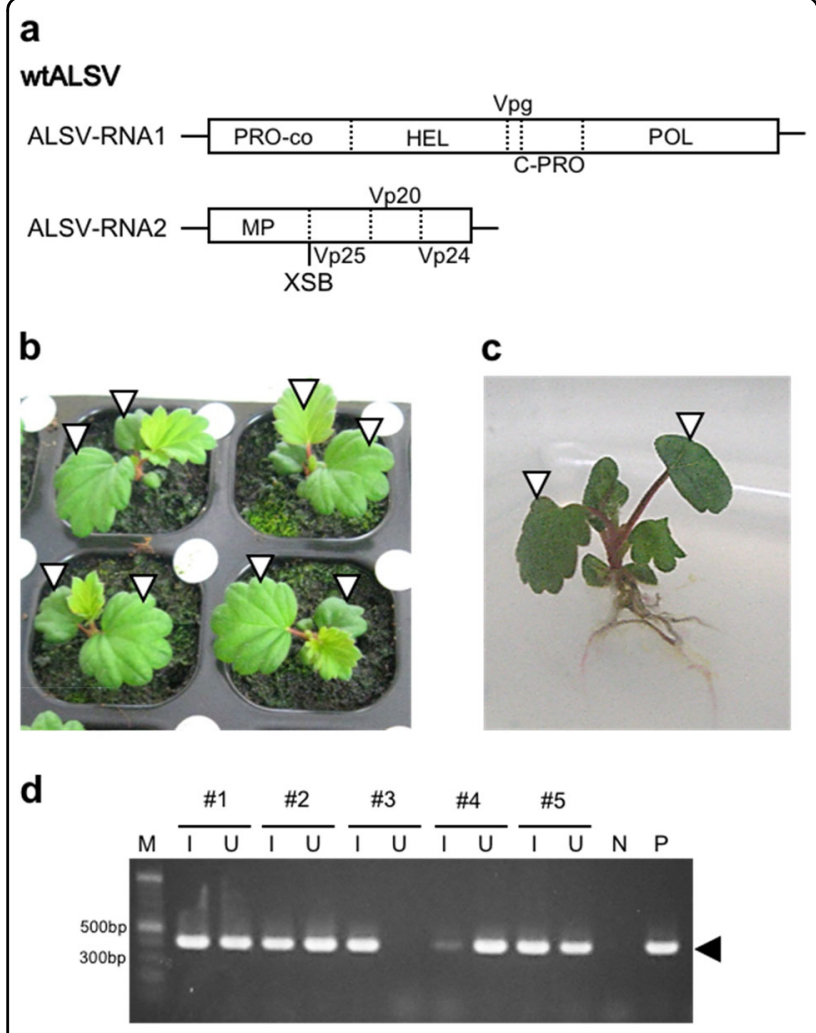

Fig. 1 Inoculation of wtALSV to strawberry seedlings. a Structure of the wtALSV. RNA1 genome encoding protease cofactor (PRO-CO), NTP-binding helicase (HEL), viral protein genome-linked (Vpg), cysteine protease (C-PRO) and RNA polymerase (POL). The RNA2 genome encodes movement protein (MP), and capsid proteins Vp25, Vp20 and Vp24. An Xhol-Smal-BamHI (XSB) cloning site was inserted in the plasmid clone of the RNA2 genome, between MP and Vp25. b Strawberry seedlings grown on soil in a plug tray. c Strawberry seedling aseptically grown on solid medium. Open arrowheads in b and $\mathbf{c}$ indicate expanded true leaves in which gold particles were bombarded. d RT-PCR assay of ALSV infection to strawberry. \#1 through \#5 indicate independent strawberry seedlings grown in vitro and inoculated with wTALSV. Symbols, I and U, indicate inoculated leaves and uninoculated upper leaves, respectively. Non-inoculated strawberry plant $(\mathrm{N})$ and wtALSV-infected Chenopodium quinoa plant (P) were also analysed as controls

ALSV-AtFT (infection rate of 58\%; Table 1). The infected plants were transferred to soil in plastic pots. Some of the infected plants set floral buds in the plant box and other plants flowered after transfer to soil in plastic pots, being self-pollinated (Fig. 3b). The size of plants infected with ALSV-AtFT, was smaller than that of mock-inoculated plants, because of the transition from the vegetative to the reproductive phase (Fig. 3c). However, fruits were mature approximately 1 month after flowering and seeds exhibited germination ability (Fig. 3d). Thus, strawberry fruits were mature 4-5 months after sowing in this analysis and seeds could be collected from mature fruits. Strawberry plants without virus infection, or plants infected by
wtALSV, did not flower or bear fruit within these time frames.

\section{ALSV distribution in reproductive organs}

Observation of photo-bleaching by VIGS indicated penetration of ALSV to many of the cells in vegetative tissues, at least in the upper part of strawberry plants. ALSV distribution in reproductive organs was also estimated by microscopic observation of viral RNA following in situ hybridization analysis. Here, reproductive organs were prepared by infection of ALSV-AtFT to induce early flowering. Sections of fixed tissues were labelled with an RNA probe. The probe was further detected using an antibody, the phosphatase activity of which was used for chromogenic reaction to generate blue indigo/formazan pigmentation. When anther tissues were labelled with an ALSV probe, blue pigmentation was observed around the endothecium and in the majority of pollen grains (Fig. 4a, top). In contrast, no pigmentation was detected when anther tissues were labelled with a control SMV probe (Fig. 4a, bottom). Similarly, blue pigmentation was observed in the ovary and ovule of immature fruits using an ALSV prove (Fig. 4b, left), whereas there was no pigmentation in any part of the fruits with an SMV probe (Fig. 4b, right). Thus, ALSV was distributed in the reproductive organs (pollen grain and ovule) in strawberry flowers.

\section{Virus elimination in progeny plants}

ALSV is transmitted to progeny plants (seed transmission), at the rates of 0 to $>50 \%$ depending on plant species. To examine possible ALSV transmission to progeny strawberry plants, next-generation plants were grown from strawberry seeds that were collected from earlyflowered parents generated by infection of ALSV-AtFT. At 2.5 months after sowing on soil (Fig. 5a), upper leaves were tested by RT-PCR or RT loop-mediated isothermal amplification (RT-LAMP). A sum of 58 independent plants was tested by either RT-PCR or RT-LAMP, respectively, with lack of amplification signal in the former, and lack of green fluorescence of calcein in the latter (Fig. 5b), indicating the absence of ALSV in progeny plants. Thus, ALSV was not detected in any of these 116 progeny plants examined (seed transmission rate of $0 \%$ ).

\section{Discussion}

In our initial trial of ALSV inoculation to juvenile strawberry seedlings by particle bombardment, wtALSV never infected to soil-cultivated plants, whereas wtALSV infected to $89 \%$ of seedlings grown in vitro (Fig. 1; Table 1). As a similar trend of difference in infection rate was also observed in ALSV infection to grapevine (Maeda et al., unpublished data), a general difference in physiological states between soil-cultivated plants and tissue- 
Table 1 Infection rates of ALSV vectors to strawberry seedling ('Yotsuboshi') and in vitro culture ('Dover')

\begin{tabular}{lllll}
\hline Cultivar & Vector & Growth condition & Growth stage at inoculation & Infected plants/inoculated plants (\%) \\
\hline 'Yotsuboshi' & wtALSV & soil (plug tray) & $3-4$ true leaves & $0 / 16(0)$ \\
'Yotsuboshi' & WtALSV & $1 / 2$ MS aseptically & $3-4$ true leaves & $8 / 9(89)$ \\
'Yotsuboshi' & ALSV-FaPDS & $1 / 2$ MS aseptically & $3-4$ true leaves & $12 / 12(100)$ \\
'Yotsuboshi' & ALSV-AtFT & $1 / 2$ MS aseptically & $3-4$ true leaves & $7 / 12(58)$ \\
'Dover' & wtALSV & $1 / 2$ MS aseptically & $3-4$ true leaves & $5 / 8(63)$ \\
'Dover' & ALSV-FaPDS & $1 / 2$ MS aseptically & $3-4$ true leaves & $5 / 5(100)$ \\
\hline
\end{tabular}

\section{a}

ALSV-FaPDS

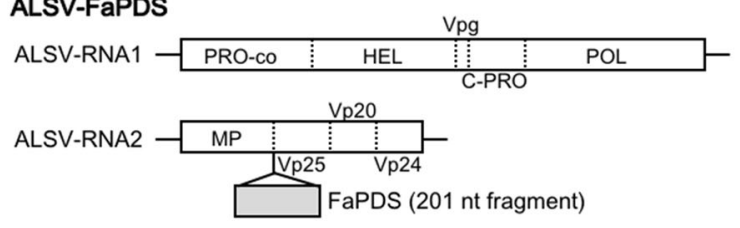

b
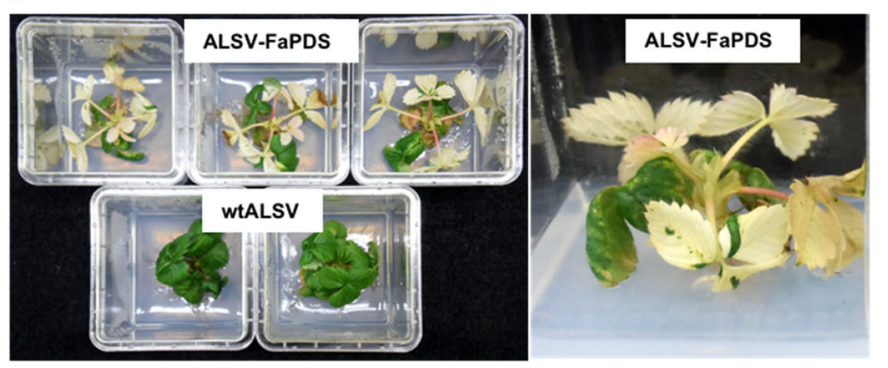

C

Fig. 2 Virus-induced gene silencing in strawberry seedling ('Yotsuboshi') and a cultivar ('Dover') grown in vitro and infected with ALSVFaPDS. a Structure of ALSV-FaPDS. b Strawberry seedlings ('Yotsuboshi') grown in vitro and infected with ALSV-FaPDS (left-top) or wtALSV (leftbottom). Photograph was taken at 45 days post inoculation (dpi). Right picture shows magnification of the representative plant. c Photo-bleaching phenotype of 'Dover' infected with ALSV-FaPDS (30 dpi)

cultured plants may exist that renders in vitro plants more susceptible to viral infection. Namai et al. ${ }^{40}$ reported that the resistance to anthracnose of tissue-cultured strawberry plants increased as the acclimation period increased, suggesting that the resistance of a strawberry cultivar may be induced by external factors. Considering that plants are equipped with molecular defense systems against virus infection $^{41}$, possible environmental effects on these defense systems, such as RNA silencing mechanism, should be examined in future studies.

The infection rate of ALSV-FaPDS to seedlings grown in vitro was $100 \%$, a similar rate as obtained with wtALSV. Conversely, infection rate of ALSV-AtFT in in vitro strawberry was only $58 \%$ (Table 1 ). Such variation in infection rate of ALSV has been repeatedly observed in

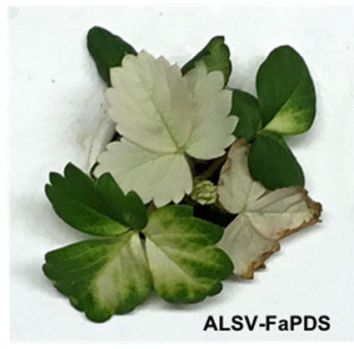

ALSV-FaPDS

many plant species. The infection rate of ALSV is affected (lowered) according to the size and nucleotide sequence of the insertion. However, insertions of $201 \mathrm{nt}$ rarely affect infection rate. The infection rate of ALSV-AtFT is expected to be sufficiently high for basic studies and applications in strawberry. Moreover, in addition to strawberry seedling ('Yotsuboshi'), successful infection of ALSV vectors was also achieved in strawberry cultivar ('Dover') propagated from in vitro culture (Table 1; Fig. 2). This suggests that ALSV technology might be applied to the analysis of gene function and promotion of flowering in the majority of strawberry cultivars.

Photo-bleaching in whole leaves, petioles and stems in the upper part of vegetative organs (Fig. 2) indicated ALSV penetration throughout the upper portions of 

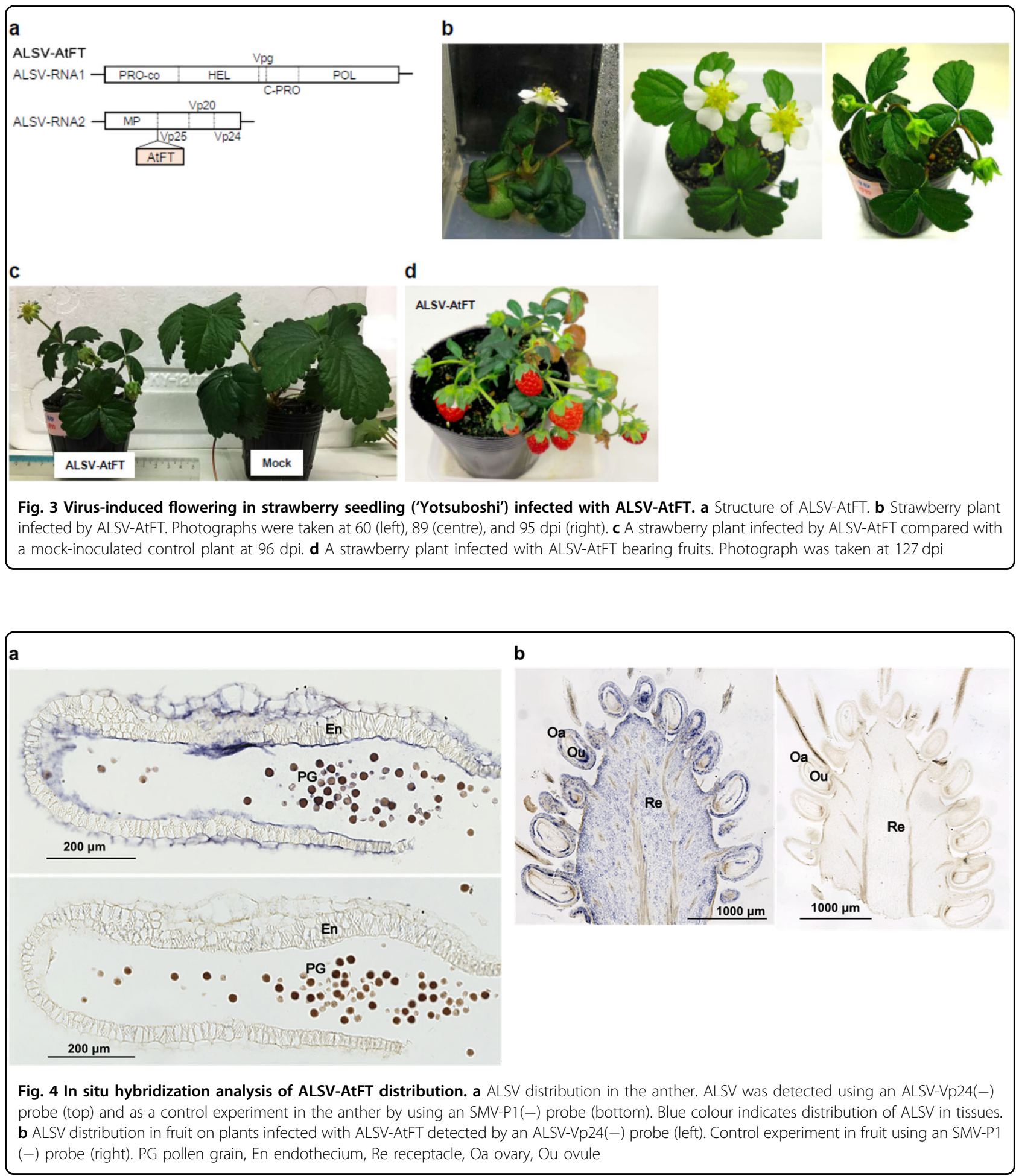

vegetative organs. In addition, systemic infection of wtALSV did not cause viral symptoms in strawberry. These results are consistent with our previous experiments in other plant species such as apple, gentian, soybean and tobacco ${ }^{35,39,42}$. In addition, analysis of ALSV infection in reproductive organs (Fig. 4) demonstrated that ALSV could be detected in many parts of pollen grains and ovules, as well as in ovaries, receptacles, petals and sepals, indicating that ALSV also penetrated reproductive organs.

As described in the Introduction, TRV vector is already reported to induce gene silencing as the sections within 

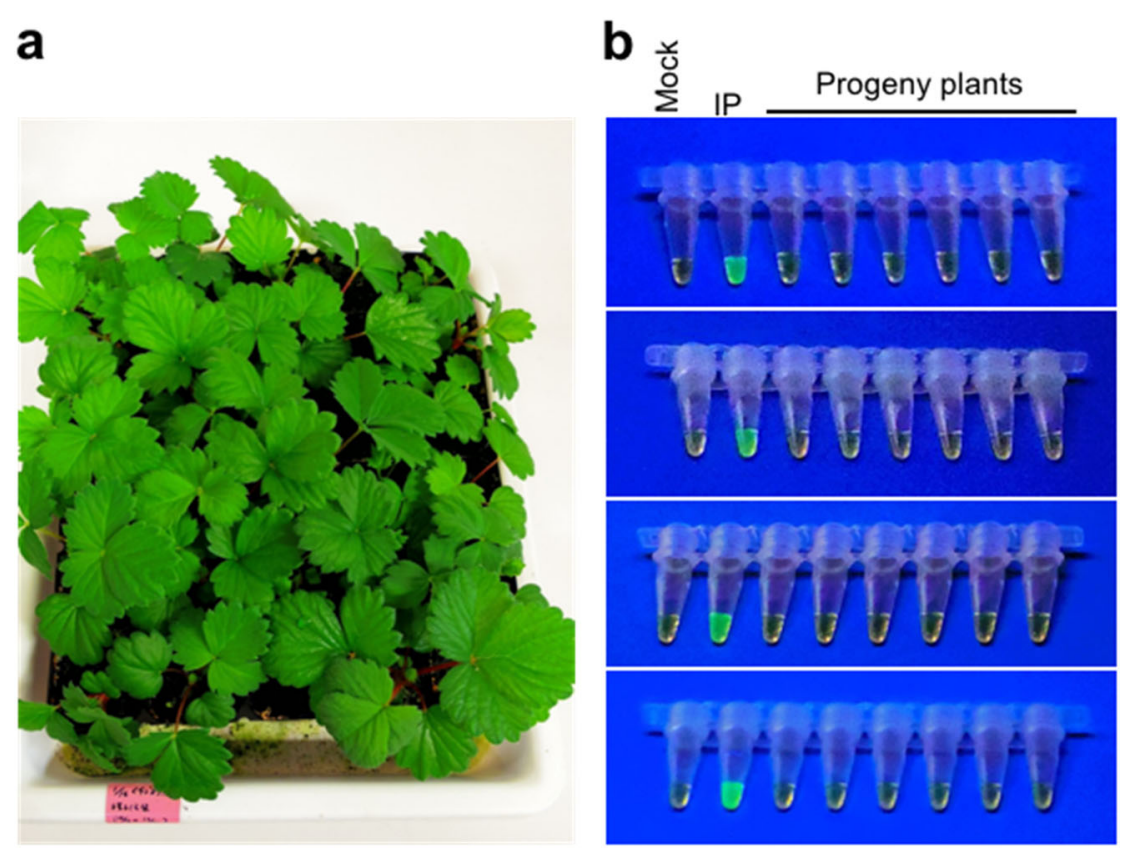

Fig. 5 Estimation of ALSV infection in progeny plants from early-flowered plants. a Progeny seedlings from early-flowered strawberry through infection of ALSV-AtFT. Photograph was taken 2.5 months after sowing. b RT-LAMP analysis of progeny plants. 'Mock' represents reaction without template DNA/RNA. IP, infected plants.

strawberry fruits ${ }^{19,20}$. The advantages of TRV compared with ALSV are that the experimental procedure is quite simple, and it can be used for VIGS study in strawberry fruits. On the other hand, the advantages of ALSV would be that ALSV can be used for gene silencing in whole vegetative tissues (leaves and stems) of strawberry shoot: ALSV induces even (overall) gene silencing in stems and 'remote' upper leaves (Fig. 2). It is not examined yet whether ALSV is applicable to VIGS in strawberry fruits, although there will be a good chance to induce even gene silencing in vegetative tissues. What is more, ALSV can induce VIF to shorten the generation time of strawberry. In practical use of ALSV for VIF of strawberry, normally grown cultivars will be crossed with each other. The $F_{1}$ seeds are gathered, aseptically cultured, and inoculated with ALSV to induce early flowering for estimation of fruit quality or further crossing. After elimination of ALSV, this virus will no longer affect flowering pattern or vegetative growth (plant size).

ALSV should preferably be removed in the next generation, when early flowering (VIF) technology is utilized for the promotion of cross-breeding. In this sense, VIF with ALSV vectors is especially convenient in gentian and lisianthus, in which ALSV is not transmitted to the next generation $^{35,43}$. In the case of apple, ALSV is transferred from the seed parent to progeny plants at a low rate $(0-4.5 \%)^{44}$. However, when we examined strawberry plants (Fig. 5), ALSV was never transferred to the next generation, suggesting that ALSV infecting pollen grains and ovules becomes degraded in strawberry embryos after pollination.

Shorter generation time is also advantageous for the generation of hybrid strawberry cultivars. Compared with traditional vegetative propagation using runners, seed-propagated characteristics are expected to ease the labour required for preparing new seedlings. To develop new hybrid cultivars, genomes of both parents are fixed to homozygotes through repetitive self-pollinations. After breeding of strawberry cultivars by using VIF technology, the absence of ALSV in progeny plants are ascertained by methods like RT-LAMP, before they are taken to open fields. Notably, such strawberry plants are not transgenic at least on the product base $e^{45-47}$, because ALSV, an RNA virus, only transiently infects strawberry cells. Our findings indicate that it is now possible to breed new strawberry cultivars by using ALSV-based VIF.

\section{Materials and methods \\ Plant materials}

Strawberry (Fragaria $\times$ ananassa Duchesne) cultivars 'Yotsuboshi' and 'Dover' were used in this study. 'Yotsuboshi' is an everbearing, seed-propagated $F_{1}$ hybrid cultivar generated by crossing between 'Mie strain 1' (seed parent) and 'A8S4-147' (pollen parent). Seeds of 'Yotsuboshi' were gathered in a naturally lit greenhouse at the 
Science and Technology Promotion Centre of Mie Prefectural Government, Japan. Strawberry seeds were sown on soil in plug trays and grown in an incubator at $25^{\circ} \mathrm{C}$, in a $16 \mathrm{~h} / 8 \mathrm{~h}$ light/dark scheme at the photosynthetic photon flux density of $150 \mu \mathrm{mol} \mathrm{m} \mathrm{m}^{-2} \mathrm{~s}^{-1}$. Alternatively, strawberry seeds were surface-sterilized in a solution containing $0.01 \%$ Contaminon (a detergent; Fujifilm-Wako, Osaka, Japan) and $0.01 \%$ sodium hypochlorite for $5 \mathrm{~min}$. Seeds were rinsed with sterilized distilled water three times, sown on half-strength MS medium solidified with $0.7 \%$ agar $^{48}$, and then grown in the incubator. Cultivar 'Dover' was also grown on the same medium. One month after inoculation of viral RNA, strawberry plants in plug trays or medium were transferred to soil in plastic pots and grown in the incubator or in a naturally lit greenhouse.

\section{Construction of the ALSV vectors}

Plasmid vectors pCALSR1 and pCALSR2, which encode the RNA1 and RNA2 genomes of ALSV, respectively, were used for the preparation of ALSV vectors ${ }^{49}$. The wtALSV vector (Fig. 1a) was prepared using pCALSR1 and pCALSR2 without any insertion sequence. For preparation of ALSV-FaPDS vector, a $201 \mathrm{nt}$ fragment of strawberry FaPDS (FJ795342, nt 1469-1669) was artificially synthesized and introduced into the plasmid (Fasmac, Atsugi, Japan). This fragment was amplified with the Xho-FaPDS $(+)$ primer (5'-CCG CTC GAG GAT TCA GAA ATT GAT GCC-3) and BamH-FaPDS(-) primer (5'-CGC GGA TCC GTC TCC AGT TAA ATA GAA ACC-3) to attach XhoI and BamHI sites at either end of the fragment, using KOD Plus Neo DNA polymerase (Toyobo, Osaka, Japan). PCR was performed at $98^{\circ} \mathrm{C}$ for $1 \mathrm{~min}$, followed by 30 cycles of denaturation at $98^{\circ} \mathrm{C}$ for $30 \mathrm{~s}$, annealing at $55^{\circ} \mathrm{C}$ for $30 \mathrm{~s}$, and extension at $68^{\circ} \mathrm{C}$ for $1 \mathrm{~min}$. The reaction was finished with an additional extension at $68^{\circ} \mathrm{C}$ for $3 \mathrm{~min}$. This PCR product was introduced into the XSB site of pCALSR2 (RNA2 genome) after digestion with XhoI and BamHI, so that the fragmental PDS codons were in frame with the codons of the RNA2 genome. For preparation of the ALSV-AtFT vector, the full-length AtFT coding sequence without the stop codon ${ }^{34}$ was introduced into the XSB site of pCALSR2 in-frame, after digestion with XhoI and BamHI.

\section{Preparation of viral RNA}

Viral RNA was prepared as previously described ${ }^{48}$. In summary, pCALSR1 and pCALSR2 plasmids were independently introduced into A. tumefaciens strain GV3101::pMP90. Nicotiana benthamiana leaves were inoculated by a 1:1:1 mixture of Agrobacterium culture transformed with pCALSR1, pCALSR2 or a transient expression plasmid encoding the HC-Pro silencing suppressor of clover yellow vein virus ${ }^{36}$. One month after inoculation, upper leaves were tested by RT-PCR for viral infection. Infected leaves were ground with buffer and rub inoculated to Chenopodium quinoa leaves. ALSV was concentrated by homogenization of infected Chenopodium leaves and treatment with bentonite solution. Viral RNA was obtained by using phenol-chloroform (1:1) extraction from this solution and precipitated with ethanol. RNA concentration was estimated by ultraviolet (UV) absorbance at $260 \mathrm{~nm}$, and stored at $-80^{\circ} \mathrm{C}$.

\section{Inoculation of viral RNA to strawberry}

Gold particles $(0.6 \mu \mathrm{m})$ (Bio-Rad Laboratories, Hercules, CA, USA) were coated with viral RNA as described previously ${ }^{38}$. For each bombardment of wtALSV, $7 \mu$ g of RNA was used, whereas $10 \mu \mathrm{g}$ of RNA was used for bombardment of ALSV-FaPDS or ALSVAtFT. Strawberry plants generated 3-4 true leaves at 1 month after sowing. The two expanded true leaves were inoculated by bombardment of RNA-coated gold particles by using a GDS-80 gene gun system (NepaGene, Ichikawa, Japan). Two shots of gold particles were used for each leaf, at the pressure of 20 psi. Gold particles are visible on leaves as brown coloration after successful bombardment.

\section{RNA extraction from strawberry}

The procedure of total RNA extraction was slightly modified from a previous report ${ }^{50}$. Strawberry leaves (half of one leaf) were excised from the tip of the uppermost compound leaf and collected in $2 \mathrm{~mL}$ plastic tubes together with two stainless beads (SUB-50, $\phi 4.8 \mathrm{~mm}$, Tomy, Tokyo, Japan), and frozen at $-80^{\circ} \mathrm{C}$ for at least $1 \mathrm{~h}$. Leaves were crushed by using a MicroSmash MS-100R (Tomy) at $2500 \mathrm{rpm}$ for $30 \mathrm{~s}$. Tubes were briefly centrifuged, and $750 \mu \mathrm{L}$ of RNA extraction buffer $(0.1 \mathrm{M}$ Tris- $\mathrm{HCl}, \mathrm{pH}$ 8.0; $25 \mathrm{mM}$ ethylenediaminetetraacetic acid, $\mathrm{pH} 8.0 ; 2 \mathrm{M}$ $\mathrm{NaCl} ; 2 \%$ cetyltrimethylammonium bromide, $2 \%$ polyvinylpyrrolidone; and 2\% 2-mercaptoethanol) was added. Tubes were mixed again by using MicroSmash at 3000 $\mathrm{rpm}$ for $30 \mathrm{~s}$, then incubated at $65^{\circ} \mathrm{C}$ for $20 \mathrm{~min}$, followed by the addition of $750 \mu \mathrm{L}$ chloroform, vortexed for $2 \mathrm{~min}$ and centrifuged at $7827 \times g$ for $10 \mathrm{~min}$ at $4{ }^{\circ} \mathrm{C}$. A $720 \mu \mathrm{L}$ fraction of the upper (water) phase was recovered to a new $1.5 \mathrm{~mL}$ tube, to which $240 \mu \mathrm{L} \mathrm{LiCl}(7.5 \mathrm{M})$ was added, mixed thoroughly and placed at $-80^{\circ} \mathrm{C}$ for at least $20 \mathrm{~min}$. RNA was precipitated by centrifugation at $15,342 \times g$ for $50 \mathrm{~min}$ at $4{ }^{\circ} \mathrm{C}$, the supernatant was discarded, and the pellet rinsed with $1 \mathrm{~mL}$ of $80 \%$ ethanol and centrifuged at $15,342 \times g$ for $5 \mathrm{~min}$ at $4{ }^{\circ} \mathrm{C}$. The supernatant was discarded, the pellet was dried and sterilized distilled water was added to dissolve the pellet. RNA concentration was estimated by UV absorbance at $260 \mathrm{~nm}$. 


\section{Reverse transcription-polymerase chain reaction}

Total RNA $(1 \mu \mathrm{g})$ was reverse transcribed with oligo$(\mathrm{dT})_{20}$ primer, by using ReverTra Ace reverse transcriptase (Toyobo). The reaction was performed at $42{ }^{\circ} \mathrm{C}$ for $60 \mathrm{~min}$, terminated at $99^{\circ} \mathrm{C}$ for $5 \mathrm{~min}$ and held at $4{ }^{\circ} \mathrm{C}$. Resultant cDNA (complementary DNA) was amplified by PCR as follows. The cDNA sample was amplified using ALSR2-999(+) (5'-GCT CTC TGT AGT TAT TCT GCA G-3) and ALSR2-1437(-) (5'-GAC CTT CTA GCA GAT TTG GG-3) primers with Ex Taq DNA polymerase (TaKaRa, Kusatsu, Japan). The reaction was performed at $95^{\circ} \mathrm{C}$ for $1 \mathrm{~min}$, followed by 40 cycles of denaturation at $94^{\circ} \mathrm{C}$ for $20 \mathrm{~s}$, annealing at $55^{\circ} \mathrm{C}$ for $20 \mathrm{~s}$ and extension at $72^{\circ} \mathrm{C}$ for $30 \mathrm{~s}$. This PCR product was electrophoresed in a $1.5 \%$ agarose gel.

\section{Reverse transcription loop-mediated isothermal amplification}

A protocol for detection of ALSV-RNA2 by RT-LAMP was recently developed for estimation of ALSV infection to gentian plants ${ }^{43}$. This protocol was followed to estimate ALSV infection to strawberry plants in the present study. Strawberry leaves were pricked with a wooden toothpick five times, and then the tip of the toothpick was rinsed in $100 \mu \mathrm{L}$ extraction buffer (Tris- $\mathrm{HCl}, \mathrm{pH} 8.0,100$ $\mathrm{mM})$. Next, $2 \mu \mathrm{L}$ of this solution was applied to a $25 \mu \mathrm{L}$ total reaction volume. RT-LAMP reaction was performed by using the Loopamp RNA Amplification Kit (Eiken Chemical, Tokyo, Japan) at $63{ }^{\circ} \mathrm{C}$ for $60 \mathrm{~min}$. The LAMP primers for ALSV detection were FIP (5'-GAA GTG GCA CTC TTA GTT GGT AAA TTG ATT ACC TAA ATA GTT GCA ATG G-3), BIP (5'-CAG GCC AGG TCA GGA TTT TGA CTA GGT GTA ACC AGC TTT G-3), F3 (5'-GCA AAC AAT ACG TGG GAA G-3) and B3 (5'AGA GAA AAA GAA AAG GAC TCA A-3). Primer concentrations in the reaction were $1.5 \mu \mathrm{M}$ for FIP and BIP, and $0.2 \mu \mathrm{M}$ for F3 and B3. RT-LAMP amplification was detected by the green fluorescence of calcein (included in the Fluorescent Detection Reagent, Eiken Chemical) emitted when reaction tubes were directly illuminated by UV.

\section{In situ hybridization}

The in situ hybridization analysis for detection of the RNA of ALSV in strawberry tissues was performed following a previously described protocol ${ }^{44}$. In summary, immature strawberry fruits (including sepals and receptacles) were fixed in FAA solution (formaldehyde alcohol acetic acid; formalin:ethanol:acetic acid:water $=$ 10:50:5:35, $\mathrm{vv}^{-1}$ ), dehydrated by serial concentrations of ethanol and lemozol, and embedded in Paraplast Plus (Sigma-Aldrich, St. Louis, MO, USA). Sections prepared at $12 \mu \mathrm{m}$ thickness were extended on APS-coated glass slides (Matsunami Glass, Osaka, Japan), deparaffinized and dehydrated. Slides were treated with proteinase K, refixed and a digoxigenin (DIG)-labelled probe that was complementary to the Vp24 region (nt 2619-3617) of the RNA2 of ALSV was hybridized; alternatively, a DIGlabelled probe complementary to the P1 region (nt 132-1045) of soybean mosaic virus was hybridized as a negative control. Slides were rinsed, then probes were labelled with sheep anti-DIG Conjugated Alkaline Phosphatase (Roche, Basel, Switzerland), rinsed again and stained with chromogenic substrate NBT/BCIP (Roche) to generate dark blue indigo and formazan dyes. The reaction was stopped by dipping the slide in water. Slides were dehydrated, dried and mounted in Entellan New (Merck, Darmstadt, Germany).

\section{Acknowledgements \\ We are grateful to Dr. Toshiki Mori, Mie Prefectural Agricultural Research Institute, Matsuzaka 515-2316, Mie, Japan, for supplying strawberry seeds of 'Yotusuboshi', and Dr. Kiyosi Namai and Ms. Keiko Nakazawa, Tochigi Prefectural Agricultural Experiment Station, Utsunomiya 320-0002, Tochigi, Japan, for supplying in vitro culture of strawberry 'Dover'. We also thank Ms. Mioko Kawasaki for assistance with virus inoculation to strawberry plants, and Ms. Motoko Sato for assistance in culturing/growing strawberry plants. This study was supported partly by the Science and Technology Research \\ Promotion Program for Agriculture, Forestry, Fisheries and Food Industry.}

\section{Authors' contributions}

C.L. and N.Y. performed VIGS and VIF experiments, respectively. I.K. and N.Y. designed the study and wrote the article.

Conflict of interest

The authors declare that they have no conflict of interest.

\section{Publisher's note}

Springer Nature remains neutral with regard to jurisdictional claims in published maps and institutional affiliations.

Received: 31 July 2018 Revised: 22 September 2018 Accepted: 28 October 2018

Published online: 01 February 2019

References

1. Yara UK Ltd. World Strawberry Production. URL: http://www.yara.co.uk/ crop-nutrition/crops/soft-fruit/key-facts/world-production/. Accessed 13 May 2018.

2. Faedi, W., Mourgues, F. \& Rosati, C. Strawberry breeding and varieties: situation and perspectives. Acta Hortic. 567, 51-59 (2002).

3. Iwata, $\mathrm{H}$. et al. The TFL 1 homologue $K S N$ is a regulator of continuous flowering in rose and strawberry. Plant J. 69, 116-125 (2012).

4. Zorrilla-Fontanesi, Y. et al. Genetic analysis of strawberry fruit aroma and identification of $\mathrm{O}$-methyltransferase FaOMT as the locus controlling natural variation in mesifurane content. Plant Physiol. 159, 851-870 (2012).

5. Zorrila-Fontanesi, Y. et al. Quantitative trait loci and underlying candidate genes controlling agronomical and fruit quality traits in octoploid strawberry (Fragaria $\times$ ananassa). Theor. Appl. Genet. 123, 755-778 (2011).

6. Lerceteau-Köhler, E. et al. Genetic dissection of fruit quality traits in the octoploid cultivated strawberry highlights the role of homoeo-QTL in their control. Theor. Appl. Genet. 124, 1059-1077 (2012).

7. Samad, S. et al. Additive QTLs on three chromosomes control flowering time in woodland strawberny (Fragaria vesca L.). Hortic. Res. 4, 17020 (2017).

8. Verma, S. et al. Clarifying sub-genomic positions of QTLs for flowering habit and fruit quality in U.S. strawberry (Fragaria $\times$ ananassa) breeding populations using pedigree-based QTLanalysis. Hortic. Res. 4, 17062 (2017). 
9. Shulaev, V. et al. The genome of woodland strawberry (Fragaria vesca). Nat. Genet. 43, 109-116 (2011).

10. Peace, C. P. DNA-informed breeding of rosaceous crops: promises, progress and prospects. Hortic. Res. 4, 17006 (2017).

11. Nehra, N. S. et al. Agrobacterium-mediated transformation of strawberry calli and recovery of transgenic plants. Plant Cell Rep. 9, 10-13 (1990).

12. Nehra, N. S. et al. Genetic transformation of strawberry by Agrobacterium tumefaciens using a leaf disk regeneration system. Plant Cell Rep. 9, 293-298 (1990).

13. Ricardo, V. G., Coll, Y., Castagnaro, A. \& Ricci, J. C. Transformation of a strawberry cultivar using a modified regeneration medium. HortScience 38, 277-280 (2003).

14. Schaart, J. G. Agrobacterium-mediated transformation of strawberry. BioProtocol 4, e1022 (2014).

15. Zakaria, H., Hussein, G. M., Abdel-Hadi, A. H. \& Abdallah, N. A. Improved regeneration and transformation protocols for three strawberry cultivars. GM Crops Food 5, 27-35 (2014).

16. Haddadi, F., Aziz, M. A., Abdullah, S. N., Tan, S. G. \& Kamaladini, H. An efficient Agrobacterium-mediated transformation of strawberry cv. Camarosa by a dual plasmid system. Molecules 20, 3647-3666 (2015).

17. Spolaore, S., Trainotti, L. \& Casadoro, G. A simple protocol for transient gene expression in ripe fleshy fruit mediated by Agrobacterium. J. Exp. Bot. 52 845-850 (2001).

18. Hoffmann, T., Kalinowski, G. \& Schwab, W. RNAi-induced silencing of gene expression in strawberry fruit (Fragaria $\times$ ananassa) by agroinfiltration: a rapid assay for gene function analysis. Plant J. 48, 818-826 (2006).

19. Chai, Y., Jia, H., Li, C., Dong, Q. \& Shen, Y. FaPYR1 is involved in strawberry fruit ripening. J. Exp. Bot. 62, 5079-5089 (2011).

20. Jia, $H$. et al. Type $2 C$ protein phosphatase $A B \mid 1$ is a negative regulator of strawberry fruit ripening. J. Exp. Bot. 64, 1677-1687 (2013).

21. Guidarelli, M. \& Baraldi, E. Transient transformation meets gene function discovery: the strawberry fruit case. Front. Plant Sci. 6, 444 (2015).

22. Cui, M. Y. et al. A rapid and efficient Agrobacterium-mediated transient gene expression system for strawberry leaves and the study of disease resistance proteins. Plant Cell Tiss. Organ Cult. 131, 233-246 (2017).

23. Hasan, O. \& Reid, J. B. Reduction of generation time in Eucalyptus globulus. Plant Growth Regul. 17, 53-60 (1995).

24. Mudge, K., Janick, J., Scofield, S. \& Goldschmidt, E. E. A history of grafting. Hortic. Rev. 35, 437-493 (2009).

25. van Nocker, S. \& Gardiner, S. E. Breeding better cultivars, faster: applications of new technologies for the rapid deployment of superior horticultural tree crops. Hortic. Res. 1, 14022 (2014)

26. Collard, B. C. et al. Revisiting rice breeding methods-evaluating the use of rapid generation advance (RGA) for routine rice breeding. Plant Prod. Sci. 20 337-352 (2017).

27. Kotoda, N., Iwanami, H., Takahashi, S. \& Abe, K. Antisense expression of MdTFL1, a TFL 1-like gene, reduces the juvenile phase in apple. J. Am. Soc. Hort. Sci. 131, 74-81 (2006).

28. Klocko, A. L. et al. FT overexpression induces precocious flowering and normal reproductive development in Eucalyptus. Eucalyptus Plant Biotechnol. J. 14, 808-819 (2016).

29. McGarry, R. C., Klocko, A. L., Pang, M., Strauss, S. H. \& Ayre, B. G. Virus-induced flowering: an application of reproductive biology to benefit plant research and breeding. Plant Physiol. 173, 47-55 (2017).

30. Lin, M. K. et al. FLOWREING LOCUS T protein may act as the long-distance florigenic signal in the cucurbits. Plant Cell 19, 1488-1506 (2007).

31. McGarry, R. C. \& Ayre, B. G. Geminivirus-mediated delivery of florigen promotes determinate growth in aerial organs and uncouples flowering from photoperiod in cotton. PLOS ONE 7, e36746 (2012).
32. Velázquez, K. et al. Precocious flowering of juvenile citrus induced by a viral vector based on Citrus leaf blotch virus: a new tool for genetics and breeding. Plant Biotechnol. J. 14, 1976-1985 (2016).

33. Yamagishi, $\mathrm{N}$. et al. Promotion of flowering and reduction of a generation time in apple seedlings by ectopical expression of the Arabidopsis thaliana FT gene using the Apple latent spherical virus vector. Plant Mol. Biol. 75, 193-204 (2011).

34. Yamagishi, N. \& Yoshikawa, N. Expression of FLOWERING LOCUS T from Arabidopsis thaliana induces precocious flowering in soybean irrespective of maturity group and stem growth habit. Planta 233, 561-568 (2011).

35. Fekih, R., Yamagishi, N. \& Yoshikawa, N. Apple latent spherical virus vectorinduced flowering for shortening the juvenile phase in Japanese gentian and lisianthus plants. Planta 244, 203-214 (2016).

36. Kasajima, I., Li, C., Yamagishi, N. \& Yoshikawa, N. ALSV vector substantially shortens generation time of horticultural plants. in Plant Engineering (ed Jurić, S.) 69-88 (IntechOpen, 2017a).

37. Yamagishi, N., Kishigami, R. \& Yoshikawa, N. Reduced generation time of apple seedlings to within a year by means of a plant virus vector: a new plantbreeding technique with no transmission of genetic modification to the next generation. Plant Biotechnol. J. 12, 60-68 (2014).

38. Kasajima, I., Ito, M., Yamagishi, N. \& Yoshikawa, N. Apple latent spherical virus (ALSV) vector as a tool for reverse genetic studies and non-transgenic breeding of a variety of crops. in Plant Epigenetics. RNA Technologies (eds Rajewsky, N., Jurga, S. \& Barciszewski, J.) 513-536 (Springer International Publishing AG, 2017b).

39. Igarashi, A. et al. Apple latent spherical virus vectors for reliable and effective virus-induced gene silencing among a broad range of plants including tobacco, tomato, Arabidopsis thaliana, cucurbits, and legumes. Virology 386, 407-416 (2009).

40. Namai, K., Matsushima, Y., Morishima, M., Amagai, M. \& Natusaki, T. Resistance to anthracnose is decreased by tissue culture but increased with longer acclimation in the resistant strawberry cultivar. J. Gen. Plant Pathol. 79, 402-411 (2013).

41. Moon, J. Y. \& Park, J. M. Cross-talk in viral defense signaling in plants. Front. Microbiol. 7, 2068 (2016).

42. Li, C., Sasaki, N., Isogai, M. \& Yoshikawa, N. Stable expression of foreign proteins in herbaceous and apple plants using apple latent spherical virus RNA2 vectors. Arch. Virol. 149, 1541-1558 (2004).

43. Kamada, K., Omata, S., Yamagishi, N., Kasajima, I. \& Yoshikawa, N. Gentian (Gentiana triflora) prevents transmission of apple latent spherical virus (ALSV) vector to progeny seeds. Planta https://doi.org/10.1007/s00425-018-2992-9 (2018).

44. Nakamura, K. et al. Seed and pollen transmission of Apple latent spherical virus in apple. J. Gen. Plant Pathol. 77, 48-53 (2011).

45. Araki, M. \& Ishii, T. Towards social acceptance of plant breeding by genome editing. Trends Plant. Sci. 20, 145-149 (2015).

46. Sprink, T., Eriksson, D., Schiemann, J. \& Hartung, F. Regulatory hurdles for genome editing: process- vs. product-based approaches in different regulatory contexts. Plant Cell Rep. 35, 1493-1506 (2016).

47. Ishii, T. \& Araki, M. A future scenario of the global regulatory landscape regarding genome-edited crops. GM Crops Food 8, 44-56 (2017).

48. Murashige, T. \& Skoog, F. A revised medium for rapid growth and bio assays with tobacco tissue cultures. Physiol. Plant. 15, 473-497 (1962).

49. Kon, T. \& Yoshikawa, N. Induction and maintenance of DNA methylation in plant promoter sequences by apple latent spherical virus-induced transcriptional gene silencing. Front. Microbiol. 5, 595 (2014).

50. Sasaki, S., Yamagishi, N. \& Yoshikawa, N. Efficient virus-induced gene silencing in apple, pear, and Japanese pear using Apple latent spherical virus vectors. Plant Methods 7, 15 (2011). 